\title{
To screen or not to screen: identification of domestic violence in Canadian emergency departments
}

\author{
Kathleen Mackay, MSW, RSW
}

See related article on Page 325

VERSION fRANÇAISE À LA PAGE 331

$T_{0}$ The study by McClennan and colleagues in this issue of CJEM, "Caring for Victims of Intimate Partner Violence: A Survey of Canadian Emergency Departments," brings needed attention to an ongoing and serious public health issue. Intimate partner violence (IPV) is a subset of family violence that includes elder abuse and child abuse, and affects the health of millions of North Americans, particularly women, each year. Despite the large magnitude of the problem of IPV, the authors found no significant increase in the existence of emergency department (ED) IPV policies over a 10-year period, which included the 2003 release of the Canadian Task Force on Preventive Health Care guidelines. Although the authors note that because of the lack of evidence for the effectiveness of interventions, task forces in the United States and Canada have not recommended for or against screening for IPV, they indicate that it is likely beneficial that health care providers identify IPV and respond in a way that maximizes benefits and avoids additional harm to the victim.

Notwithstanding our general ambivalence for universal IPV screening, it is worth noting that there are important advantages that may arise from such an intervention. Based on my experience over the last 15 years as the coordinator of domestic violence (DV) programs in 2 major teaching hospitals and a DV outpatient clinic, I have found that ED visits represent opportunities to promote awareness of the health impact of IPV, to ask questions about patients' safety in relationships, and to provide information on hospital and community resources to help victims and abusers.

The DV program at Vancouver General Hospital was one of the first in Canada. It commenced in 1992 with a grant from Health Canada. The goal was to develop a program of care to identify, assess, treat, refer and follow up with women who presented to the ED with IPV. Universal screening of all female ED patients by nurses was implemented, with specific protocols for care and documentation by the interdisciplinary team when IPV was disclosed. Previously, our data indicated that very few IPV cases were identified in the ED. Since the launch of the program, thousands of patients experiencing IPV have been asked about abuse, listened to, validated, encouraged to access resources and provided with documentation. We now screen all adults, male or female, throughout Providence Health Care, which includes St. Paul's Hospital and Mount Saint Joseph's Hospital, and in the ED and Pre-Admission Clinic at Vancouver General Hospital.

The Vancouver General Hospital DV program and those like it promote health by increasing awareness of the health impact of IPV and offering early intervention to those affected. By asking about abuse, seeds of hope are planted in those who deny abuse, and some return at a later date for assistance. Others who are asked may have friends or family members in abusive situations and can gain valuable knowledge about where abused people can go for help. In this respect, simply asking is a potentially beneficial intervention in itself. Despite this, screening for IPV remains controversial. The causes and possible interventions in IPV are complex. As such, it is perhaps not surprising that task forces in the United States and Canada have, to date, not identified specific interventions with 
proven effectiveness, and thus have been unable to recommend for or against screening for IPV.

IPV is a multifaceted health and social problem and involves another individual, the abuser, whose actions cannot be controlled. Although the primary focus of IPV intervention is to increase the safety of the abused, numerous media accounts underscore the fact that an abuser who is motivated to harm or even kill his estranged partner or family members often succeeds.

For decades, community agencies have assisted victims of domestic violence and administered programs for perpetrators. Health agencies, in contrast, have been slow to respond to this issue. McClennan and colleagues ${ }^{1}$ suggest that implementing policies and procedures that respond to the need of patients who are exposed to IPV should be a priority. I could not agree more. However, let us not wait for the impossible - a one-fits-all intervention that is proven to be effective and can therefore justify screening for IPV. There is simply no panacea intervention that will bring an end to this perplexing health, social and community ill.

In many respects IPV is analogous to the issue of drunk driving, where public perception and community standards can influence behaviour. Media attention on the victim impact of drunk driving has encouraged a number of strategies, including more responsible drinking, designated drivers and the use of taxis. Family violence has not had the benefit of sustained public awareness campaigns targeted at reducing abuser behaviour. Most abusers do not appreciate the profound impact their actions have on others. We cannot begin to solve the problem of IPV until abuser behaviour is systematically addressed. Public awareness about the nature of domestic abuse and its impact on the family is a critical part of that solution. I believe there is an essential role for our EDs in this effort, at a minimum by displaying posters and providing information on abuse and community resources for victims and perpetrators. Although the benefits remain unproven, I also believe universal screening for victimization is appropriate in the ED. The victim is in the ED already, and is likely to come again if she or he continues to be abused. Identifying the problem, validating the abused as not being at fault, encouraging safety behaviours and documenting the situations are all feasible in the brief period of time during the patient encounter. This is potentially very cost-effective because the system is already seeing the patient and the intervention is predominately one of empathy and providing information. Asking about IPV opens the door to invaluable healing and may help reduce morbidity and mortality from this serious public health issue.

\section{Competing interests: None declared.}

Keywords: domestic violence, intimate partner violence, emergency department, screening, public awareness

\section{References}

1. McClennan S, Worster A, MacMillan H. Caring for victims of intimate partner violence: a survey of Canadian emergency departments. CJEM 2008;10:325-8.

Correspondence to: Ms. Kathleen Mackay, Domestic Violence Program,Vancouver General Hospital, 855 West 12th Ave., Vancouver BC V5Z1M9; kathleen.mackay@vch.ca

\section{Erratum}

A CAEP Update about the Canadian Emergency Department Triage and Acuity Scale (CTAS) adult guidelines in the March 2008 issue contained an error. Box 1 should have listed the classification of bleeding severity for moderate, minor bleeds as Level III, not Level II. We regret the error.

\section{Reference}

1. Bullard MJ, Unger B, Spence, J, et al; the CTAS National Working Group. Revisions to the Canadian Emergency Department Triage and Acuity Scale (CTAS) adult guidelines. CJEM;10:136-42. 